\section{International Scientific Journal Theoretical \& Applied Science}

\author{
p-ISSN: 2308-4944 (print) e-ISSN: 2409-0085 (online) \\ Year: 2015 Issue: 10 Volume: 30
}

Published: $30.10 .2015 \quad$ http://T-Science.org

SECTION 21. Pedagogy. Psychology. Innovations in the field of education.
Said Abdullaevich Salekhov

Professor, doctor of medical sciences Novgorod State University of Yaroslav Mudry, Russia ssalehov@mail.ru

Michail Nickolaevich Gordeev Professor, doctor of medical sciences, Institute of Psychotherapy and Clinical Psychology

Russia

mngordeev@yandex.ru

Tatiana Vladimirovna Bizina graduate student, Novgorod State University of Yaroslav Mudry, Russia tatyana-bizina@yandex.ru

\title{
INFLUENCE OF PRE-EXAMINATIONAL CORRECTION OF SITUATION ANXIETY ON EXAM RESULTS
}

\begin{abstract}
The dynamics of the situation anxiety test results were studied in the group of 148 second year Medical College female students. The tests took place during the preliminary exam which had been hold for 3 days before the exam in biochemistry and then during the exam itself. The participants were divided into two groups depending on the targeted psychological assistance to decrease psycho-emotional tension before the exams. The first group of 67 students didn't get any psycho-emotional aid. The second group of 81 students were provided with psycho-emotional compensation according to our algorithm, which considered the psycho-physiological features of emotional tension increase and its energy demand. Before the preliminary exam the situation anxiety indices correlated and didn't differ much $(P>0,05)$. On the contrary, before the exam the number of the second group students who showed an average level of situation anxiety were credibly more $(P<0,05)$, and those who showed high level were credibly less $(P<0,05)$ then in the first group, where no psychological aid had been provided. At the same time the average score showing the situation anxiety distinctiveness before the exam was credibly higher in the first group $(P<0,05)$, then in the second, that proved the effectiveness of the psycho-emotional compensation program. The comparative analysis of the preliminary exam and the exam itself and the dynamics of the situation anxiety showed that the psycho-emotional tension increase caused by the exam stress created the prerequisites for the exam results in the first group. In the second group the dynamics of the situation anxiety testified of the psychoemotional tension increase before the exam, but the degree of its distinctiveness was credibly less, then in the first group. At that the results of the exam tests in the second group were higher than those of the first group, that was caused by less psycho-emotional tension before the exam due to the psychological assistance in the second group for 3 days before the exam. The results prove the availability of the following research in this field.

Key words: exam stress, psycho-emotional tension, situation anxiety, psychological assistance, psychoemotional compensation

Language: Russian

Citation: Salekhov SA, Gordeev MN, Bizina TV (2015) INFLUENCE OF PRE-EXAMINATIONAL CORRECTION OF SITUATION ANXIETY ON EXAM RESULTS. ISJ Theoretical \& Applied Science 10 (30): 117-123.

Soi: http://s-o-i.org/1.1/TAS-10-30-25 Doi: crossef http://dx.doi.org/10.15863/TAS.2015.10.30.25

\section{ВЛИЯНИЕ ПРЕДЭКЗАМЕНАЦИОННОЙ КОРРЕКЦИИ СИТУАТИВНОЙ ТРЕВОЖНОСТИ НА РЕЗУЛЬТАТЫ ЭКЗАМЕНОВ}

Аннотация: Была исследована динамика результатов исследования ситуативной тревожности у 148 студенток 2 курса медицинского института перед предварительным, проводивиимся за 3 суток перед экзаменом, и экзаменационным тестированием по биохимии. В зависимости от проводимого целенаправленного психологического сопровождения, направленного на снижение психоэмочиионального напряжения перед экзаменами, участниц исследования разделили на 2 группьы. В I группу вошли 67 студенток, которым коррекция психоэмоционального состояния не проводилась. Во II группу вошла 81 студентка, у которых после предварительного тестирования в течение 3 дней 2 раза в день проводилась коррекция психоэмоционального состояния в соответствии с разработанным нами алгоритмом, учитывающего психофизиологические особенности развития эмоционального напряжения и его
\end{abstract}


энергетического обеспечения. Перед предварительным тестированием показатели ситуативной тревожности в исследуемых группах были сопоставимыми и достоверно не различались $(P>0,05)$. B отличие от этого, перед экзаменом во II группе число студенток со средним уровнем ситуативной тревожности было достоверно больше $(P<0,05)$, а с высоким уровнем достоверно меньше $(P<0,05)$ чем 6 I, где челенаправленного психологического сопровождения не проводилось. При этом средний балл, отражаюший выраженность ситуативной тревожности перед экзаменом в I группе был достоверно больше $(P<0,05)$, чем во II, что свидетельствовало об эффективности проводимых в ней мероприятий по коррекции психоэмоционального состояния. Сравнительный анализ результатов предварительного и экзаменационного тестирования с динамикой ситуативной тревожности перед их проведением показал, что на фоне значительного нарастания психоэмочионального напряжения, обусловленного экзаменационным стрессом, создавались предпосылки для снижения экзаменационных показателей в I группе. Во II группе динамика ситуативной тревожности свидетельствовала о возрастании психоэмочионального напряжения перед экзаменом, но степень его выраженности было достоверно меньше, чем в I группе. При этом на фоне меньшего психоэмочиионального напряжения перед экзаменами, что было обусловлено проводимой психологическим сопровождением II группы в течение 3 суток, предшествующих экзамену, результаты экзаменащионного тестирования во II группе достоверно превысили как показатели, зарегистрированные в I группе. Полученные результаты свидетельствуют о перспективности дальнейтих исследований в этом направлении.

Ключевые слова: экзаменачионный стресс, психоэмочиональное напряжение, ситуативная тревожность, психологическое сопровождение, коррекция эмоичионального напряжения.

Психологический стресс стал символом современного образа жизни, поскольку на смену реальных угрожающих жизни ситуаций пришли ситуации, сопровождающиеся интенсивным психоэмоциональным напряжением и необходимостью обработки и реагирования на лавинообразно возрастающий поток информации. То есть, ситуации, которые мы воспринимаем как стрессовые, и на которые реагируем соответственно, реагируем на уровне всего организма в соответствии с активизацией рефлекса «борьба-бегство» [1, С. 88-131]. Этот диагноз стал широко распространенным и огульно применяемым при нарушении психологического и физического состояния [2, С. 326; 3, С. 7].

Академический стресс, являющийся разновидностью психологического стресса, позволяет наиболее полно оценить воздействие стрессоров на студентов, особенно в период адаптации к обучению в колледже, либо высшем учебном заведении (ВУЗ). При этом на учащихся одновременно влияют изменения условий проживания, переезд на новое место жительства, само проживание в условиях общежития, построение отношений в новом коллективе в группе, с профессорско-преподавательским составом учебного заведения, в общежитии, изменение экономических условий, необходимость самостоятельного принятия решений и ответственности за них. Список можно продолжить и значительно расширить.

Следует отметить, что все эти изменения образа жизни формируют психоэмоциональный фон, на который наслаиваются резко возрастающий объем информации для обработки и усвоения, а также дополнительные воздействия стрессовых факторов во время экзаменов. При этом экзаменационный стресс (ЭС), особенно в период адаптации на 1-2 курсах обучения в ВУЗе, является одним из наиболее ярких и значимых явлений, определяющих психоэмоциональное и когнитивное состояние учащихся.

Экзамен, отличающийся высокой когнитивной нагрузкой в условиях ограниченного времени на фоне интенсивного психоэмоционального напряжения и неопределенностью конечного результата, требует внутренней мобилизации студента на личностно-мотивационном, эмоциональноволевом и индивидуально-типологическом уровнях. Соответственно, на фоне экзаменационного стресса создаются предпосылки для нарушения функциональных возможностей памяти и мышления, нарастания тревожности и страха, снижения настроения, внимания, самооценки и работоспособности в целом [3, С. 35-43; 4, Р. 255-256; 5, S. 67-68].

Способам саморегуляции психологического состояния в стрессовых ситуациях и экзаменационного стресса в частности уделяется пристальное внимание, о чем свидетельствует растущее число публикаций, посвященных этой тематике [3, С. 154-199; 6, 176 с.; 7, с. 278-282; 8, Р. 181-187; 9, Р. 44; 10, Р. 105; 11, Р. 15]. При этом особое место в коррекции психоэмоционального состояния играет регуляция дыхания, что применяется как во время медитаций, так и самостоятельно [3, С. 170-171; 12, С. 113-122; 13].

Следует отметить, что существует большое число вариантов психологического сопровождения во время экзаменационного периода, которые, даже при комплексном подходе, носят эмпирический характер, без научного обоснования этапов непосредственно 
перед экзаменом и экзаменационного тестирования. При этом не учитываются иерархия психоэмоционального напряжения и резко возрастающей когнитивной нагрузки, что достаточно часто приводит к снижению оценки знаний студентов.

Соответственно, разработка и внедрение в предэкзаменационную и экзаменационную подготовку новых методов психологической коррекции, направленной на повышение результативности экзаменационного тестирования, является перспективным направлением научных исследований.

\section{Материалы и методы исследования}

В основу данной публикации положен анализ динамики результатов исследования ситуативной тревожности по шкале STAI перед предварительным компьютерным тестированием и через 3 суток накануне экзамена по биологической химии у 148 студенток 2 курса медицинского института во время зимних экзаменационных сессий 2012-2015 г.г. Критерием значимости и информативности шкалы STAI являлось сопоставление результатов предэкзаменационного и экзаменационного тестирования в зависимости от проводимого психологического сопровождения в течение 3 суток, предшествующих экзамену.

Шкала тревоги Спилбергера (State-Trait Anxiety Inventory - STAI) является информативным способом самооценки уровня тревожности в данный момент (реактивная тревожность, как состояние) и личностной тревожности (как устойчивая характеристика человека).

Ситуативная или реактивная тревожность как состояние характеризуется субъективно переживаемыми эмоциями: напряжением, беспокойством, озабоченностью, нервозностью. Это состояние возникает как эмоциональная реакция на стрессовую ситуацию и может быть разным по интенсивности и динамичности во времени. Личности, относимые к категории высоко тревожных, склонны воспринимать угрозу своей самооценке и жизнедеятельности в обширном диапазоне ситуаций и реагировать весьма выраженным состоянием тревожности. Состояние реактивной (ситуационной) тревоги возникает при попадании в стрессовую ситуацию и характеризуется субъективным дискомфортом, напряженностью, беспокойством и вегетативным возбуждением. Естественно, это состояние отличается неустойчивостью во времени и различной интенсивностью в зависимости от силы воздействия стрессовой ситуации [14, с.104$108 ; 15$, с. 3-38].

Таким образом, значение итогового показателя по шкале ситуативной тревожности позволяет оценить не только уровень актуальной тревоги испытуемого, но и определить, находится ли он под воздействием стрессовой ситуации, какова интенсивность этого воздействия и насколько она влияет на его состояние.

Все студентки, рассматриваемые в данной работе, дали письменное информированное согласие на участие в исследовании. Исследование проводилось вечером за 3 суток до экзамена и, непосредственно, накануне экзамена.

Студентки в перименструальном периоде, перенесшие в течение года какие либо тяжелые соматические заболевания (вирусный гепатит, пневмония, отравления), операции и травмы в настоящем исследовании не рассматривались.

Следует отметить, что отсутствие должного психологического сопровождения студенток в течение семестра и во время приближающейся экзаменационной сессии создавало предпосылки для выраженности ЭС во время сессии и его влияния на результаты успеваемости.

Критериями включения в программу исследования являлись результаты предыдущих сессий на первом курсе, когда различия между количеством правильных ответов на предварительном и экзаменационном тестировании было $10 \%$ и более. что привело к снижению итоговой оценки, при хорошей академической успеваемости в течение межсессионного периода.

В зависимости от проводимой коррекции психоэмоционального состояния были образованы 2 группы.

В I группу вошли 67 студенток, которым коррекция психоэмоционального состояния не проводилась.

Во II группу вошла 81 студентка, у которых после предварительного тестирования в течение 3 дней 2 раза в день проводилась коррекция психоэмоционального состояния в соответствии с разработанным нами алгоритмом, учитывающего психофизиологические особенности развития эмоционального напряжения и его энергетического обеспечения.

Для коррекции психоэмоционального состояния во время подготовки к экзаменам был предложен алгоритм, включающий:

1. Дыхательные техники, предусматривающие эпизоды задержки дыхания, что обеспечит снижение интенсивности психоэмоционального напряжения. При задержке дыхания развивается кратковременная гипоксия, на фоне которой происходит торможение доминантного очага возбуждения в лимбической системе, поддерживающего психоэмоциональное напряжение, что создает предпосылки для восстановления регуляторной функции центральной нервной системы (ЦНС).

$$
\text { 2. Только после снижения }
$$
психоэмоционального напряжения 
обеспечивается поступление в организм углеводов легко всасывающихся в пищеварительном тракте, что обеспечит полноценное обеспечение высшей нервной деятельности энергоносителями (в первую очередь глюкозой) и кислородом.

3. Обучение экспресс-технике/ам (дыхание в режиме вдох - задержка - выдох) снижения психоэмоционального напряжения с учетом физиологических и психофизиологических особенностей энергообеспечения высшей нервной деятельности.

4. Применение техник НЛП и/или эриксоновского гипноза для формирования нового восприятия экзамена. В нашей работе мы использовали технику НЛП - генератор нового поведения [16, с. 139-146] и ресурсные трансы [17, с. 186-192].

5. Обеспечение полноценного режима подготовки, чередования умственной и физической активности, полноценный ночной сон во время предэкзаменационной подготовки и непосредственно, перед экзаменом.

Соблюдение последовательности приведенного алгоритма создает предпосылки для управления психоэмоциональным состоянием и восстановление приоритетности когнитивной функции ЦНС.

Bce студентки исследуемых групп проживали в благоустроенном общежитии, были не замужем и не имели детей. При этом социальные и бытовые условия в исследуемых группах были схожими. Возраст студенток I группы составил 20,2 $\pm 0,6$ лет, а во II - 19,8 $\pm 0,4$ года, при этом достоверность различий между исследуемыми группами отсутствовала (Р>0,05).

Учитывая, что участницами исследования были студентки медицинского института, при обучении экспресс-методам обретения спокойного состояния создавались дополнительные благоприятные условия и возможности использования когнитивного восприятия информации для понимания механизмов управления психоэмоциональным состоянием на физиологическом и биохимическом уровне как в процессе обучения, так и, особенно, в экстремальной ситуации, например, в режиме экзаменационного тестирования.

Статистическую обработку полученных результатов производили по Стьюденту, рассчитывали среднюю арифметическую (М), ошибку средней арифметической (m) и достоверность различий $(\mathrm{P}<0,05)$ по формуле и таблицам Стьюдента.

\section{Результаты исследования}

Анализ динамики результатов исследования ситуативной тревожности (шкала STAI) перед предварительным тестированием и накануне экзамена показал, что отмечались однотипные отрицательные изменения всех исследуемых показателей как в I (контрольной), так и II (основной) группах. При этом интерес представляли как изменения происходящих изменений в каждой группе, так и сравнительный анализ полученных результатов в исследуемых группах между собой (Табл. 1).

Сравнительный анализ динамики ситуативной тревожности в I группе показал, что накануне экзамена отмечалось достоверное изменение выраженности ее количественных и качественных характеристик $(\mathrm{P}<0,05)$.

Так, было выявлено увеличение ситуативной тревожности, что подтверждалось ее переходом из среднего уровня в высокий у $62,7 \pm 5,9 \%$ (Р $<0,05)$. При этом высокий уровень ситуативной тревожности был выявлен у всех участниц исследования I группы, что достоверно превышало показатели, зарегистрированные перед предварительным тестированием $(\mathrm{P}<0,05)$. Более того, перед экзаменом средний балл ситуативной тревожности в I группе в 1,7 раз превышал показатели, зарегистрированные накануне предварительного тестирования (Р $<0,05)$.

Таблица 1 Динамика ситуативной тревожности в исследуемых группах перед предварительным тестированием и экзаменом.

\begin{tabular}{|c|c|c|c|c|}
\hline \multirow[t]{2}{*}{ Исследуемые показатели } & \multicolumn{2}{|c|}{ I группа (n=67) } & \multicolumn{2}{|c|}{ II группа (n=81) } \\
\hline & Предварит. & Экзамен & Предварит. & Экзамен \\
\hline Низкая & - & - & - & - \\
\hline $\begin{array}{c}\text { Средняя: } \\
\text { Абс. кол-во } \\
\text { М } \pm \mathbf{m}(\%)\end{array}$ & $\begin{array}{c}42 \\
62,7 \pm 5,9\end{array}$ & - & $\begin{array}{c}46 \\
56,8 \pm 5,5\end{array}$ & $\begin{array}{c}11 \\
13,6 \pm 3,8^{*, \Delta}\end{array}$ \\
\hline $\begin{array}{c}\text { Высокая } \\
\text { Абс. кол-во } \\
\text { М } \pm \mathbf{m}(\%)\end{array}$ & $\begin{array}{c}25 \\
37,3 \pm 5,9\end{array}$ & $\begin{array}{c}67 \\
100 *\end{array}$ & $\begin{array}{c}35 \\
43,2 \pm 5,5\end{array}$ & $\begin{array}{c}70 \\
86,4 \pm 3,8^{*, \Delta}\end{array}$ \\
\hline Средние баллы $(\mathrm{M} \pm \mathrm{m})$ & $38,7 \pm 3,2$ & $66,9 \pm 2,3 *$ & $42,4 \pm 3,5$ & $51,2 \pm 2,7^{\Delta}$ \\
\hline
\end{tabular}
* - достоверность различий между показателями перед предварительным и экзаменаџионным

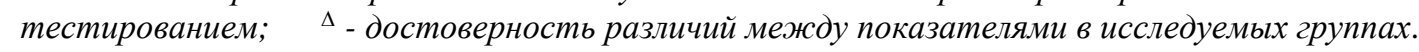


Аналогичная динамика отмечалась и во II группе, где перед экзаменом число участниц со средним уровнем ситуативной тревожности было достоверно меньше, чем перед предварительным тестированием (P <0,05). Параллельно отмечалось достоверное количество студенток с высоким уровнем ситуативной тревожности (Р $<0,05)$.

Следует отметить, что на фоне общей тенденции увеличения среднего балла перед экзаменами, по сравнению с показателями, зарегистрированными перед предварительным тестированием, изменения были недостоверными ( $\mathrm{P}>0,05)$.

Особого внимания заслуживало сравнение динамики показателей в исследуемых группах между собой. Так, если перед предварительным тестированием достоверных различий между группами выявлено не было ( $>0,05)$, то перед экзаменом во II группе число студенток со средним уровнем ситуативной тревожности было достоверно больше (P $<0,05)$, а с высоким уровнем достоверно меньше $(\mathrm{P}<0,05)$ чем в $\mathrm{I}$, где целенаправленного психологического сопровождения не проводилось. При этом средний балл, отражающий выраженность ситуативной тревожности перед экзаменом, в I группе был достоверно больше $(\mathrm{P}<0,05)$, чем во II, что свидетельствовало об эффективности проводимых в ней мероприятий по коррекции психоэмоционального состояния.

Поскольку во время предыдущих экзаменационных сессий (зимняя и летняя сессии на I курсе) у участниц исследования отмечалось снижение показателей во время экзамена по сравнению с предварительным тестированием, аналогичную динамику можно было ожидать и при сдаче экзаменов по биохимии в формате компьютерного тестирования в зимнюю сессию на II курсе. При этом оценивая динамику результатов экзамена, обращали внимание на изменение итоговой оценки как в положительную, так и отрицательную сторону.

Было установлено (Табл. 2.), что в I группе, как и ожидалось, отмечалась тенденция к снижению результативности экзаменационного тестирования по сравнению с предварительным у $70,1 \pm 5,6 \%$ участниц исследования, что достоверно превышало показатели во II, где снижение результатов было выявлено лишь у $9,9 \pm 3,3 \%(\mathrm{P}<0,05)$.

Сравнительный анализ результатов экзаменов в исследуемых группах.

Таблица 2

\begin{tabular}{|c|c|c|c|c|}
\hline \multirow{2}{*}{ Результаты экзаменов } & \multicolumn{2}{|c|}{ І группа $(\mathrm{n}=67)$} & \multicolumn{2}{|c|}{ II группа $(\mathrm{n}=81)$} \\
\cline { 2 - 5 } & Абс. кол-во & $\mathrm{M} \pm \mathrm{m}$ & Абс. кол-во & $\mathrm{M} \pm \mathrm{m}$ \\
\hline$<100 \pm 2,0 \%$ & 47 & $70,1 \pm 5,6$ & 8 & $9,9 \pm 3,3^{\Delta}$ \\
\hline $100 \pm 2,0 \%$ & 11 & $16,5 \pm 4,4$ & 18 & $22,2 \pm 4,6$ \\
\hline$>100 \pm 2,0 \%$ & 9 & $13,4 \pm 4,1$ & 55 & $67,9 \pm 5,1^{\Delta}$ \\
\hline Оценка «-» & 36 & $53,7 \pm 6,1$ & 5 & $6,2 \pm 2,6$ \\
\hline Оценка «+» & 7 & $10,4 \pm 3,6$ & 41 & $50,6 \pm 5,6^{\Delta}$ \\
\hline Средний балл (экзамен) $\mathrm{M} \pm \mathrm{m} \%$ & \multicolumn{2}{|c|}{$95,3 \pm 2,6$} & \multicolumn{2}{|c|}{$118,3 \pm 4,3^{\Delta}$} \\
\hline
\end{tabular}

${ }^{\Delta}$ - достоверность различий между исследуемыми группами.

Экзаменационные результаты совпадали с результатами предварительного тестирования в I группе у $11(16,5 \pm 4,4 \%)$ участниц, а во II - у 18 $(22,2 \pm 4,6 \%)$. При этом различия между группами были недостоверными (Р >0,05).

Особого внимания заслуживает увеличение правильных ответов на экзамене у достоверно большего количества участниц исследования (Р $<0,05)$ во II группе $(67,9 \pm 5,1 \%)$, по сравнению с I $(13,4 \pm 4,1 \%)$.

Учитывая, что итоговая оценка располагается в заданном интервале баллов, полученных на экзамене, их изменение отмечалось реже, чем увеличение, либо уменьшение числа правильных ответов. Так, в I группе у $36(53,7 \pm 6,1 \%)$ отмечалось снижение оценки во время экзамена, по сравнению по результатам предварительного тестирования, при этом во II группе снижения экзаменационной оценки отмечалось лишь у 5 (6,2 $2,6 \%$ ).

В отличие от этого, во II группе у 41 $(50,6 \pm 5,6 \%)$ студентки экзаменационная оценка была выше предварительной, в то время как в I - 
лишь у $2(8,0 \pm 5,4 \%)$. При этом различия между группами были достоверными ( $\mathrm{P}<0,05)$.

Следует отметить, что если рассматривать результаты предварительного тестирования как $100 \%$, то в I группе средний бал на экзамене составил 95,3 $\pm 2,6 \%$, в то время, как во II группе результаты были достоверно лучше $(118,3 \pm 4,3 \%)$ как показателей в I группе $(\mathrm{P}<0,05)$, так и результатов предварительного тестирования (Р $<0,05)$.

Полученные результаты свидетельствуют о том, что в I группе во время зимней сессии на втором курсе результаты предварительного тестирования и экзаменов были аналогичными показателям, зарегистрированным во время зимней и летней сессии на первом курсе. То есть, в большинстве случаев имело место снижение результативности на экзамене, по сравнению с показателями предварительного тестирования.

Сопоставив результаты предварительного и экзаменационного тестирования с динамикой ситуативной тревожности перед их проведением видно, что отмечалось значительное нарастание психоэмоционального напряжения, обусловленного ЭС, что и создавало предпосылки к снижению числа правильных ответов во время экзамена в I группе.
В отличие от этого, во II группе динамика ситуативной тревожности свидетельствовала о возрастании психоэмоционального напряжения перед экзаменом, но степень его выраженности было достоверно меньше, чем в I группе. На фоне меньшего психоэмоционального напряжения перед экзаменами, что было обусловлено проводимой психологическим сопровождением II группы в течение 3 суток, предшествующих экзамену, результаты экзаменационного тестирования во II группе достоверно превысили как показатели, зарегистрированные в I группе, так и, что особенно важно, результаты предварительного тестирования.

Таким образом, при отсутствии полноценного психологического сопровождения студентов во время учебного процесса, применение предложенной программы коррекции психоэмоционального состояния, с учетом психофизиологических особенностей реагирования на ЭС, позволили снизить его интенсивность и улучшить результаты экзаменов. Соответственно, целесообразно продолжить и расширить исследования в данном направлении.

\section{References:}

1. Grinberg JS (2002) Upravlenie stressom. S-Pb, "Piter", 2002, - 496 p. (Seriya Mastera psihologii)

2. Psihofisiologia (2006) / pod red. UI Aleksandrova .S-Pb, 2006. 331 p.

3. Scherbatih UV. (2012) Psihologia stressa I metodi korrekcii. 2 izd. - S-Pb.: Piter, 2012. $256 \mathrm{p}$.

4. Oaten M, Cheng K (2005) Academic Examination Stress Impairs Self-Control. // J. Soc Clin. Psych.: - 2005, Vol. 24, No. 2, pp. 254-279. doi: $10.1521 /$ jscp.24.2.254.62276

5. Krejnina O (2007) Negatywny stosunek do siebie jako czynnik strachu studentow przed egzaminami // Studia PsychologicznoPedagogiczne / Red.: Ju. Karandashev, T. Senko. Bielsko-Biala: Wyzsza Szkola Administracji, 2007.-Tom 1. -pp. 167-170.

6. Richardson M, Abraham C, Bond R (2012) Psychological correlates of university students' academic performance: A systematic review and meta-analysis // Psych Bul. - 2012, Vol 138(2), pp. 353-387.

7. Komarova IA (2009) Korrekcia urovnia psihoemocionalnogo stressa u studentov vo vrevia uchebnogo processa. / diss... kand. Med nauk.: Kurgan, 2009. - 178 p.

8. Kozorez EV, Kirianova EN (2012) Vlianie ekzamenacionnogo stressa na psihoemocionalnoe sostoianie obuchaushihsia / Centr psihologo-medico-socialnogo soprovojdenia // Mat. III mejdunarodnoy nauchno-prakticheskoy konf. «Psihologopedagogicheskie problem lichnosti I socialnogo vzaimodeysnvia». - 2012. - pp. 278-282.

9. Wons A, Bargiel-Matusiewicz K (2011) The emotional intelligence and coping with stress among medical students. // Wiad Lek. 2011; 64(3): 181-187.

10. Chew BH, Zain AM, Hassan F (2013) Emotional intelligence and academic performance in first and final year medical 


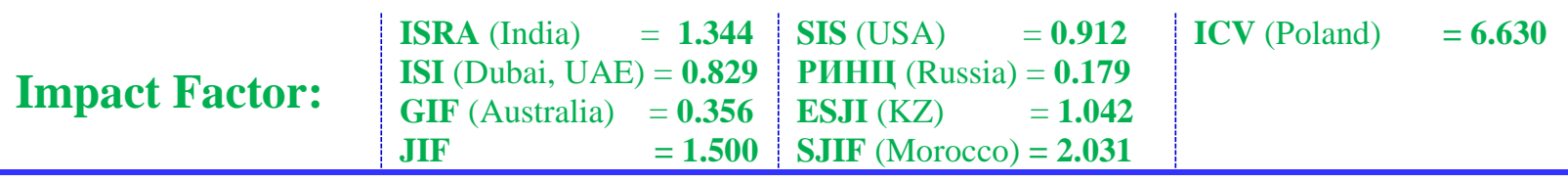

students: a cross-sectional study. // BMC Med Educ. 2013 Mar 27;13:44. doi: 10.1186/14726920-13-44

11. Bigna JJ, Fonkoue L, Tchatcho MF, et all. (2014) Association of academic performance of premedical students to satisfaction and engagement in a short training program: a cross sectional study presenting gender differences. // BMC Res Notes. 2014 Feb 24;7: 105. doi: 10.1186/1756-0500-7-105

12. Garland EL, Geschwind N, Peeters F, Wichers $M$ (2015) Mindfulness training promotes upward spirals of positive affect and cognition: multilevel and autoregressive latent trajectory modeling analyses. // Front Psychol. 2015 Feb 2;6:15. doi: 10.3389/fpsyg.2015.00015. eCollection 2015.

13. Tang YY, Hölzel BK, Posner MI (2015) The neuroscience of mindfulness meditation. //
Nature Reviews Neuroscience (2015) doi:10.1038/nrn3916 (Published online 18 March 2015).

14. Salekhov SA, Maksimyuk NN, Maratova AM (2013) Osobennostii metabolizma I psihoemocionalnogo sostoiania bobibilderov pri snijenii vesa / NovGU imeni Iaroslava Mudrogo: Velikie Novgorod, 2013. - 144 p.

15. Spielberger CD (1966) Theory and research of anxiety. // Anxiety and Behavior. : abstr. -N.Y., 1966. pp. 3-38.

16. Gordeev MN (2006) NLP v psihoterapii. «Psihoterapia». Moskva, 2006. - pp. 139-146.

17. Gordeev MN (2008) Klassicheskiy I eriksonovskiy gipnoz. «Psihoterapia». Moscow, 2008. - pp. 186-192. 